\title{
An Improved Adaptive Federal Kalman Filter Algorithm For Integrated Navigation
}

\author{
Ying Zhai ${ }^{1, a}$, Xisheng $\mathrm{Li}^{1, \mathrm{~b}}$, Yibo Feng ${ }^{1, \mathrm{c}}$, Xiaojuan Zhang ${ }^{1, \mathrm{~d}}$ \\ ${ }^{1}$ University of Science and Technology Beijing, Beijing , 100083, China

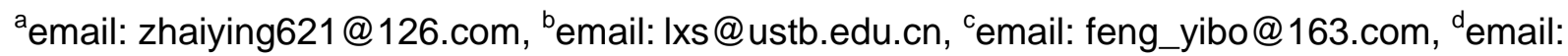 \\ zxjjianghan@163.com
}

Keywords: SINS; Integrated Navigation; GPS; Adaptive Federal Kalman Filter

\begin{abstract}
Integrated navigation system has become an important navigation system. On the basis of previous research about the federal Kalman filter, this paper mainly studied the key technologies of low-cost SINS/GPS/Magnetic compass integrated navigation system, an adaptive federal filter method of vector-form information distribution coefficients is proposed and compared with ordinary federal Kalman filter and the adaptive federal filter based on filter covariance matrix eigenvalue decomposition. The simulation results show that the proposed algorithm can get higher accuracy of navigation.
\end{abstract}

\section{Introduction}

With the development of modern technology, single navigation system is difficult to meet the needs of people. Strapdown inertial navigation system neither needs external information nor radiates any electromagnetic waves, but its error accumulates over time [1]. GPS can provides high precision navigation information all-weather and all the time, but it has a low output frequency, and it is easy to lose signals when there are lots of obstacles [2]. Compass has a wide measuring range, high stability and anti-jamming characteristics, it can provides high-precision heading information with accelerometer. However, it is easy to be disturbed by external magnetic field [3]. Therefore, SINS/GPS/Magnetic compass are integrated to increase the precision of the navigation system and meet the demands of navigation system.

Federal Kalman filter has a great advantages such as fast calculation, strong fault tolerance and convenient for the fault detection and isolation of subsystems, but it is still based on a single model of filter. With the change of system or environment, estimate error will increase and even diverge. An adaptive federal filter method of vector-form information distribution coefficients is proposed and compared with ordinary federal Kalman filter and the adaptive federal filter based on filter covariance matrix eigenvalue decomposition through navigation experiment.

\section{Principle of Adaptive Federal Kalman Filter}

In recent years, the application of Kalman filter techniques in integrated navigation system achieved great development [4]. The emergence of new technologies include: the federal extended Kalman filter, the unscented Kalman filter and the adaptive Kalman filter technique, etc [5, 6].

Federal Kalman filter theory is a kind of special form of distributed Kalman filter method, its general principle and basic design process are as seen in [7, 8]. Analysis shows that performance of federal Kalman filter is depend on sub-filter's information distribution coefficient value $\beta_{i}$ and main filter's information distribution coefficient value $\beta_{m}$. In fact, performance of navigation equipment and sub-filter may be affected by environmental factors, which will affect the estimation precision of the whole system.

In order to improve the performance of federal Kalman filter, a variety of dynamic information distribution methods are proposed, such as method based on filter covariance matrix eigenvalue decomposition, method based on observable matrix condition number [9, 10], etc. Information distribution coefficients of the above researches are based on scalar form. They treat each element 
of the state variables as the same. In fact, different sensors have different functional properties and accuracy, state equation and observation equation established by sub filters are also different.

Accordingly, existing methods with scalar distribution coefficients are difficult to timely reflect dynamic characteristics of each state variable. In view of the above situations, an adaptive federal filter method of vector-form information distribution coefficients is proposed.

1. Solution of $M_{i}$

Covariance matrix of subsystem $P_{i}$ is decomposed with characteristic values:

$$
P_{i}=L_{i} \Lambda_{i} L_{i}^{T}
$$

where $\Lambda_{i}=\operatorname{diag}\left\{\lambda_{i 1}, \lambda_{i 2}, \cdots, \lambda_{i n}\right\}, \lambda_{i 1}, \cdots, \lambda_{\text {in }}$ are the characteristic values of $P_{i}, \mathrm{n}$ is the order number of matrix $P_{i}, L_{i}$ is the matrix that decomposed by eigenvalues of matrix $P_{i}, X_{i j}$ is the j-th element of the error state in the i-th sub filter. Information distribution coefficients are calculated as follows:

$$
m_{i j}=\frac{1 / \lambda_{i j}}{1 / \lambda_{1 j}+1 / \lambda_{2 j}+\cdots 1 / \lambda_{N j}}
$$

where $i=1,2, \cdots, N, j=1,2, \cdots, n, \lambda_{i j}$ is the characteristic value that corresponded with the state variable $X_{i j}, \mathrm{~N}$ is the number of subsystem.

Information distribution coefficients matrix corresponded to $X_{i}$ is:

$$
M_{i}=\operatorname{diag}\left\{m_{i 1} m_{i 2} \cdots m_{i n}\right\}
$$

2. Solution of $\Upsilon_{i}$

If $T$ ( $T \in R^{p \times q}$ ) is the observation matrix of system, then it can be decomposed with singular values as follows:

$$
T=U S V^{T}
$$

where $U$ is the left singular matrix of $T$, and it is the orthogonal matrix with order of $p \times p$, $U=\left[u_{1} u_{2} \cdots u_{p}\right], u_{1} u_{2} \cdots u_{p}$ are the left singular column vectors. $S=\left[\begin{array}{cc}\Lambda_{r \times r} & 0 \\ 0 & 0\end{array}\right], \Lambda_{r \times r}=\operatorname{diag}\left(\sigma_{1} \sigma_{2} \cdots \sigma_{r}\right)$, $r 、 \sigma_{i}(i=1,2, \cdots, r)$ respectively represent the rank and singular values of matrix $T$. V is the right singular matrix of $T$, and it is the orthogonal matrix with order of $q \times q, V=\left[v_{1} v_{2} \cdots v_{q}\right]$, $v_{1} v_{2} \cdots v_{q}$ are the right singular column vectors.

Through the analysis of matrix $V$, this paper proposes that the right singular vector $v_{i}$ is corresponded with $\sigma_{i}$, and $\sigma_{i}$ is a singular value that corresponds with the maximum absolute values of the state variables which are gotten from $v_{i}$. From singular value decomposition of the subsystems' observation matrix, each element's information distribution coefficients of $X_{i}$ are calculated as:

$$
\Upsilon_{i j}=\left\{\begin{array}{cc}
\frac{\sigma_{i j}}{\sigma_{1 j}+\sigma_{2 j}+\cdots+\sigma_{N j}} & \sigma_{i j} \neq 0 \\
0 & \sigma_{i j}=0
\end{array}\right.
$$

where $\sigma_{i j}$ is the singular value that corresponds with the state variable $X_{i j}$, information distribution coefficients corresponded with matrix $X_{i}$ are:

$$
\Upsilon_{i}=\operatorname{diag}\left\{\gamma_{i 1} \gamma_{i 2} \cdots \gamma_{i n}\right\}
$$

3. Solution of $B_{i}$

$$
B_{i}=\frac{1}{2} \times\left(M_{i}+\Upsilon_{i}\right)=\operatorname{diag}\left\{\beta_{i 1} \beta_{i 2} \cdots \beta_{i n}\right\}
$$


Meanwhile, the federal Kalman filter information distribution coefficients satisfy the information conservation principle:

$$
\sum_{i=1}^{N} B_{i}=I_{n \times n}
$$

According to the federal filter information distribution coefficients of vector form, $Q(k)$ and $P(k)$ are distributed to each sub filter as follows:

$$
\left\{\begin{array}{l}
P_{i}^{-1}(k)=\sqrt{B_{i}} P_{f}^{-1}(k) \sqrt{B_{i}} \\
Q_{i}^{-1}(k)=\sqrt{B_{i Q}} Q_{f}^{-1}(k) \sqrt{B_{i Q}} \\
\hat{X}_{i}(k)=\hat{X}_{f}(k)
\end{array}\right.
$$

\section{System Models}

SINS has many advantages such as strong autonomy, high reliability and comprehensive output information, so it is selected as the public reference system. GPS is selected as observation systems 1 , and then SINS/GPS are combined to get the position sub filter and velocity sub filter. Magnetic compass is selected as observation systems 2, and then SINS/Magnetic compass are combined to get the posture sub filter [11]. System integration scheme is seen as figure1.

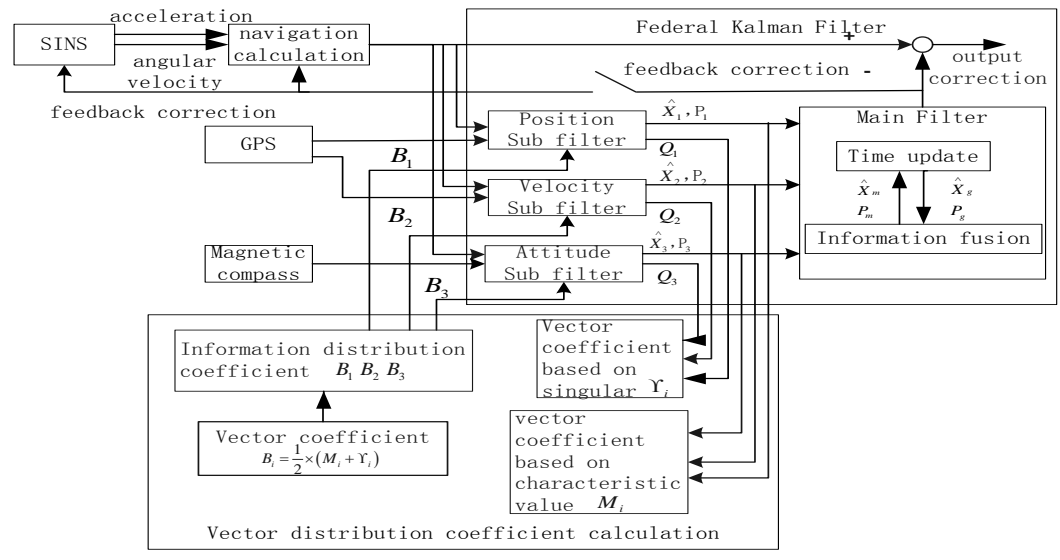

\section{1、 SINS/GPS}

Fig.1. Structure of the integrated navigation system

(1)SINS/GPS position sub filter

The state equation of position sub filter is:

$$
X_{1}=F_{1} X_{1}+G_{1} W_{1}
$$

State vector is selected as follows:

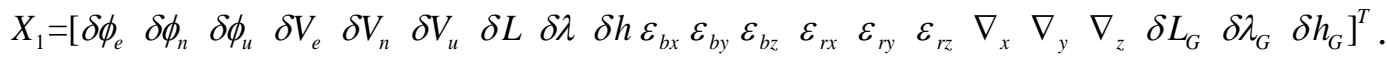

where $F_{1} 、 G_{1} 、 W_{1}$ respectively represent the state transition matrix, the noise driving matrix and the

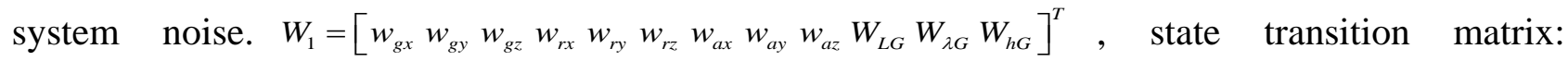
$F_{1}=\operatorname{diag}\left\{F_{I}, F_{G}\right\}, F_{G}=\operatorname{diag}\left[-\frac{1}{\tau_{L G}},-\frac{1}{\tau_{\lambda G}},-\frac{1}{\tau_{h G}}\right]$. Noise driving matrix: $G_{1}=\operatorname{diag}\left[G_{I}, I_{3 \times 3}\right]$.

The difference of position information between SINS and GPS is selected as the measurement quantity, the measurement model is:

$$
Z_{1}=\left[\begin{array}{c}
L_{I}-L_{G} \\
\lambda_{I}-\lambda_{G} \\
h_{I}-h_{G}
\end{array}\right]=H_{1} X_{1}+V_{1}
$$

where $H_{1}=\left[\begin{array}{llll}0_{3 \times 6} \operatorname{diag}\left(\mathrm{R}_{N} \cos \varphi\right. & \mathrm{R}_{M} & 1 & 0_{3 \times 9}\end{array}\right], \mathrm{V}_{1}=\left[\begin{array}{lll}N_{e} & N_{n} N_{u}\end{array}\right]^{T}$ is zero-mean white measurement noise of GPS. 
(2) SINS/GPS velocity sub filter

State vector is selected as follows:

$X_{2}=\left[\delta \phi_{e} \delta \phi_{n} \delta \phi_{u} \delta V_{e} \delta V_{n} \delta V_{u} \delta L \delta \lambda \delta h \varepsilon_{b x} \varepsilon_{b y} \varepsilon_{b z} \varepsilon_{r x} \varepsilon_{r y} \varepsilon_{r z} \nabla_{x} \nabla_{y} \nabla_{z}\right]^{T}$.

The state equation of velocity sub filter is:

$$
X_{2}=F_{2} X_{2}+G_{2} W_{2}
$$

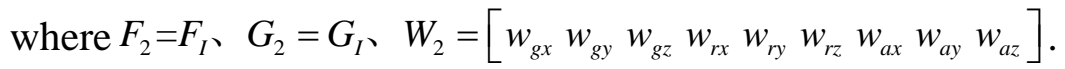

The measurement vector $Z_{2}$ is the velocity difference, which is written as:

$$
Z_{2}=\left[\begin{array}{c}
V_{I e}-V_{G e} \\
V_{I n}-V_{G n} \\
V_{I u}-V_{G u}
\end{array}\right]=H_{2} X_{2}+V_{2}
$$

where $H_{2}=\left[0_{3 \times 3} I_{3 \times 3} 0_{3 \times 12}\right], V_{1}=\left[M_{e} M_{n} M_{u}\right]^{T}$ is zero-mean white measurement noise of GPS.

2、SINS/Magnetic compass

The state equation of attitude sub filter is:

where $X_{3}=X_{2} 、 F_{3}=F_{2} 、 G_{3}=G_{2} 、 W_{3}=W_{2}$.

$$
X_{3}=F_{3} X_{3}+G_{3} W_{3}
$$

The measurement vector $Z_{3}$ is the attitude difference, which is written as:

$$
Z_{3}=\left[\begin{array}{c}
\phi_{I e}-\phi_{e e} \\
\phi_{I n}-\phi_{e n} \\
\phi_{I u}-\phi_{e u}
\end{array}\right]=H_{3} X_{3}+V_{3}
$$

where $H_{3}=\left[\begin{array}{ll}I_{3 \times 3} & 0_{3 \times 15}\end{array}\right], \mathrm{V}_{3}=\left[\begin{array}{lll}P_{e} & P_{n} & P_{u}\end{array}\right]^{T}$ is zero-mean white measurement noise of Magnetic compass.

\section{Matlab Simulation Experiment}

In order to verify the effectiveness and accuracy of the proposed algorithm, field vehicle experiment was carried out. Three filter methods are compared through MATLAB simulation. In the process of experiment, sample frequency of SINS, GPS and Magnetic compass are set to 10Hz. Filter frequency of the main filter is $1 \mathrm{~Hz}$ and the total filter time is about 35 minutes. Initial state of the system is as follows, velocity: $0 \mathrm{~m} / \mathrm{s}$, pitch angle: $-0.6354 \mathrm{deg}$, roll angle: $0.5631 \mathrm{deg}$, course angle: 89.92deg, longitude: 116.3552deg, latitude: 39.9901deg, altitude: $41.5938 \mathrm{~m}$. In order to compare the performance of the integrated navigation system, a high precision gyroscope is selected as the benchmark, and the errors between them are shown in the figures below.

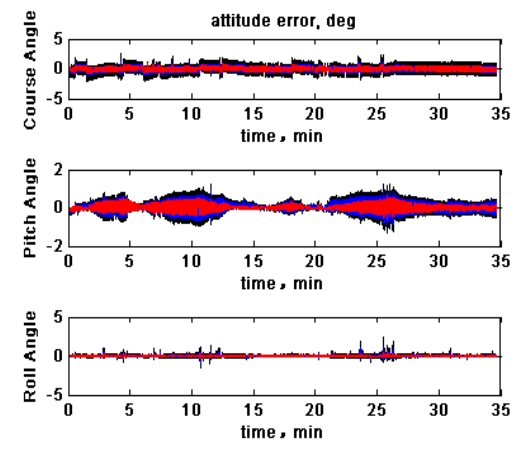

Fig.2. Attitude error
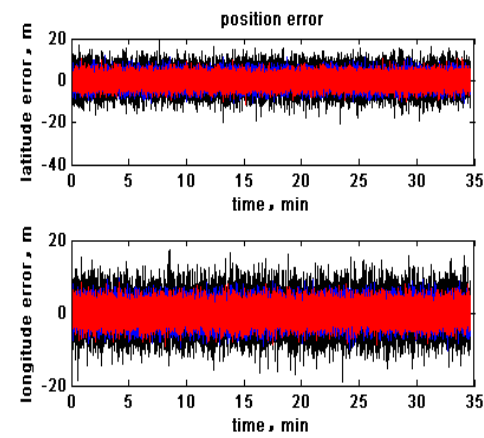

Fig.3. Position error
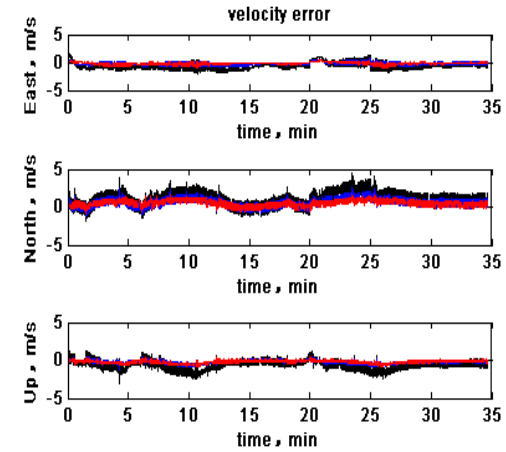

Fig.4. Velocity error

According to above figures, black line represents ordinary federal Kalman filter, blue line represents the adaptive federal filter based on filter covariance matrix eigenvalue decomposition, red line represents the adaptive federal filter method of vector-form information distribution coefficients. It is obvious that the errors of attitude, position and velocity from the proposed algorithm is more smooth and lower than ordinary federal Kalman filter. When compare the improved adaptive federal Kalman filter with the adaptive federal Kalman filter based on filter 
covariance matrix eigenvalue decomposition, the overall performance improvement is not obvious, but its convergence is better. GPS lose signals when vehicle entered into the floor shielding zone in the twentieth minute and left in the twenty-fifth minute, but the integrated navigation system is not affected by it, and still remain high navigation accuracy. It reflects a good fault tolerant performance and improves the system's dynamic response ability and anti-interference ability.

\section{Conclusion}

Inertial navigation, magnetic compass and GPS have become an important part of the integrated navigation system. An adaptive federal filter method of vector-form information distribution coefficients used in integrated navigation system can improve the system's accuracy effectively. Reliability, stability and anti-interference ability of the integrated navigation system are improved.

\section{Acknowledgement}

In this paper, the research is supported by the National Natural Science Foundation of China (Project 61273082).

\section{References}

[1] W Wu, X Ning, L Liu. New celestial assisted INS initial alignment method for lunar explorer[J]. Systems Engineering and Electronics, Journal of, 2013, 24(1): 108-117.

[2] H Qin, L Cong, X Sun. Accuracy improvement of GPS/MEMS-INS integrated navigation system during GPS signal outage for land vehicle navigation[J]. Systems Engineering and Electronics, Journal of, 2012, 23(2): 256-264.

[3] XJ Zhang, XS Li, YB Feng. Comparison of three kinds of compensation algorithms based on magnetic sensors[C]. 2013,2.675-678.

[4] Annamalai A S K, Motwani A, Sutton R, et al. Integrated navigation and control system for an uninhabited surface vehicle based on interval Kalman filtering and model predictive control[C] Control and Automation 2013: Uniting Problems and Solutions, IET Conference on. IET, 2013: 1-6.

[5] XC Yan, YZ Ouyang, FP Sun, et al. Kalman filter applied in underwater integrated navigation system[J]. 2013.

[6] K Liu, J Li, W Guo, P Zhu, et al. Navigation system of a class of underwater vehicle based on adaptive unscented Kalman fiter algorithm[J]. Journal of Central South University, 2014, 21: 550-557.

[7] MY Fu, ZH Deng, JW Zhang. Kalman filter theory and its application in the navigation system [M]. Science Press, 2003.

[8] QT Gu, S Wang. Study on the Theory of Federated Filter [J]. Journal of Chinese Inertial Technology. 2002, 10(6): 34-40.

[9] YF Wang, XL Huang, HZ Hu Heng. Eigenvalue Alue-decom position-based adaptive information fusion filter algorithm for integrated navigation systems[J]. Acta Aeronautica et Astronautica Sinica. 2000, 21(3): 274-276.

[10] G Yuan, K Yuan, H Zhang. A variable proportion adaptive federal kalman filter for INS/ESGM/GPS/DVL integrated navigation system[C]. IEEE, 2011,978-981.

[11] X Zhang, J Li, Q Jiang, et al. Design of Federal Kalman Filter Based on MIMU MIMU/GPS/EC [J]. Journal of Test and Measurement Technology. 2012, 4: 12. 